(C) 2011 IEEE. Personal use of this material is permitted. Permission from IEEE must be obtained for all other uses, in any current or future media, including reprinting/republishing this material for advertising or promotional purposes, creating new collective works, for resale or redistribution to servers or lists, or reuse of any copyrighted component of this work in other works. 


\title{
Intelligent Driver Drowsiness Detection System Using Uncorrelated Fuzzy Locality Preserving Analysis
}

\author{
Rami N. Khushaba, Sarath Kodagoda, Sara Lal, Gamini Dissanayake
}

\begin{abstract}
One of the leading causes of automobile accidents is related to driving impairment due to drowsiness. A large percentage of these accidents occur due to drivers' unawareness of the degree of impairment. An automatic detection of drowsiness levels could lead to lower accidents and hence lower fatalities. However, the significant fluctuations of the drowsiness state within a short time poses a major challenge in this problem. In response to such a challenge, we present the Uncorrelated Fuzzy Locality Preserving Analysis (UFLPA) feature projection method. The proposed UFLPA utilizes the changes in driver behavior, by means of the corresponding Electroencephalogram (EEG), Electrooculogram (EOG), and Electrocardiogram (ECG) signals to extract a set of features that can highly discriminate between the different drowsiness levels. Unlike existing methods, the proposed UFLPA takes into consideration the fuzzy nature of the input measurements while preserving the local discriminant and manifold structures of the data. Additionally, UFLPA also utilizes Singular Value Decomposition (SVD) to avoid the singularity problem and produce a set of uncorrelated features. Experiments were performed on datasets collected from thirty-one subjects participating in a simulation driving test with practical results indicating the significance of the results achieved by UFLPA of 94\%-95\% accuracy on average across all subjects.
\end{abstract}

\section{INTRODUCTION}

Driving is one of the most common attention-demanding tasks in daily life, as it requires the vehicle operator to maintain a constant level of alertness or awareness. Driver's drowsiness is a serious problem that is believed to be a direct and contributing cause of road-related accidents as it progressively impairs the driver's awareness about external events. The loss of awareness is usually associated with a reduction in perception, recognition and vehicle control abilities [1]. Recent studies have shown that driver drowsiness is believed to account for $20 \%$ of all vehicle accidents on motorways and monotonous roads ${ }^{1}$. Given the severity of this problem, developing corrective measures for the practical evaluation of the different drowsiness levels is of significant importance to prevent disastrous outcomes.

The approaches that have been used for driver's drowsiness detection can be primarily divided into three categories. The first category focus on detecting physical changes during

Rami N. Khushaba, Sarath Kodagoda, and Gamini Dissanayake are with the ARC centre of Excellence for Autonomous Systems, Faculty of Engineering and Information Technology, University of Technology, Sydney (UTS), Australia. Email: Rkhushab, sakoda, gdissadeng.uts. edu . au

Sara Lal is with the Department of Medical and Molecular Biosciences, Faculty of Science, University of Technology, Sydney (UTS), Australia. Email: Sara.Lal@uts.edu.au

${ }^{1}$ Information cited from Driver Fatigue Information fact sheet published at www.mynrma.com.au in Dec 2007 drowsiness by image processing techniques, such as average of eye-closure speed, percentage of eye-closure over time, eye tracking as quantization of drowsiness levels, and driver's head movements [2], [3]. The second category includes techniques that correlate the driver's alertness state with a set of vehicle behavioral parameters including steering wheel angular position, speed of car movement, lane deviation, and changing course [4], [5]. Several limitations of both of these categories were reported by many authors suggesting devising different methods for drowsiness detection [6], [7]. The third category includes techniques attempting to identify physiological associations between driver drowsiness and the corresponding patterns of the Electroencephalogram (EEG) (brain activity), Electrooculogram (EOG) (eye movement), and Electrocardiogram (ECG) (heart rate) signals [8], [9], [10]. Most of these studies reported that the physiological approach to drowsiness detection can provide very accurate results [6], as strong correlation between these signals and the driver's cognitive state was found in many studies. Specifically, the change in the cognitive state can be associated with significant changes in EEG frequency bands such as delta $(\delta: 0-4 \mathrm{~Hz})$, theta $(\theta: 4-8 \mathrm{~Hz})$, alpha $(\alpha: 8-13 \mathrm{~Hz})$, and beta $(\beta: 13-20 \mathrm{~Hz})[11],[12]$ or their combinations [13], or with changes in the eyelid parameters extracted from the EOG [14], and changes in the heart rate variability using the ECG power spectrum [15]. The long-term aim of this work is oriented toward an intelligent driver drowsiness detection system combining information from the aforementioned categories. However, various limitations exist in each category. In this paper, we focus on optimizing the performance of the third category using state-of-the-art feature extraction and projection so that it can complement the information extracted from other categories in a future work.

One of the main limitations in developing a practical drowsiness detection system based on physiological signals is related to the large number of channels employed within many of the above studies to capture the brain cognitive state. As an example, sixty-two EEG channels were utilized by $\mathrm{Fu}$ et al. [16] to distinguish awake, drowsy and sleep in a driving simulation study, while Lin et al. [17] utilized thirtytwo EEG/EOG channels and 2 ECG channels, Wallerius et al. [18] used 21 EEG channels, and Yeo et al. [19] used nineteen EEG channels and one EOG recording. The large number of channels was reported to be crucial for the success of many systems, as a small number of channels was reported to compromise the classification accuracy [19], [16]. The justification is that when using a small number of channels, the available feature extraction methods are not 
able to capture the complex overlapping of the different drowsiness levels. However, a small number of channels means the system is closer to a practical solution in terms of physical electrodes attachment and computational complexity of the algorithm.

On the other hand, a significant drop in classification accuracy was also reported in the literature when classifying the extracted features into three or more classes, rather than two binary classes (either awake and sleep) due to the fluctuations of the drowsiness states or levels [16]. Hence, a new dimensionality reduction/feature projection method is needed to extract an informative feature set that can best discriminate between the different drowsiness levels or states.

In this paper, a new feature projection technique that we refer to as the Uncorrelated Fuzzy Locality Preserving Analysis (UFLPA) is developed in an attempt to overcome many of the limitations of the available methods. The proposed method introduces the concept of neighborhood and locality sensitive analysis into fuzzy discriminant analysis through a theoretically justified approach. Additionally, the features generated by the proposed UFLPA are statistically uncorrelated among each other. The main goal here is to find the minimum number of uncorrelated features preserving the relevant information with minimum dimensions. This will in turn result in a classifier with fewer adaptive parameters to be determined, i.e., a classifier with better generalization properties.

The structure of this paper is as follows: Section II reviews the available dimensionality reduction methods with their associated limitations. Section III presents the proposed UFLPA method. The data collection procedure is described in section IV. Section V presents the experimental results. Finally, conclusions are given in Section VI.

\section{Feature Projection Based Dimensionality REDUCTION}

In the literature, Principal component analysis (PCA) and Linear discriminant analysis (LDA) are commonly used dimensionality reduction methods [20] (include one ref for LDA). Different from PCA, LDA is more suitable for classification problems, as it projects the data along the directions that maximize the ratio of the between-class scatter matrix $\left(S_{b}\right)$ to the within-class scatter matrix $\left(S_{w}\right)$ of the projected data. However, there are a number of limitations associated with classical Linear Discriminant Analysis (LDA) including, the need of the scatter matrix to be nonsingular and the number of features generated by LDA is bounded by the number of classes, $c-1$ (the rank of $S_{b}$ ). Both LDA and PCA can only see the global Euclidean structure of data. In order to capture the local manifold structure, many attempts were proposed in the literature, including Locality Sensitive Discriminant Analysis (LSDA) [25] and Locality Preserving Projections (LPP) [26]. However, like classical LDA, these methods require a preprocessing step (PCA or SVD) or a proper regularization method to avoid singularity [24], [27].

Given the particular problem of drowsiness detection, it is quite common to have large fluctuations in the drowsiness levels; in such a case, the local data structure might be more important than the global structure for discriminant analysis. Further, most bio-signals generated by the human body tend to generate patterns that are fuzzy in their nature, i.e., patterns that may belong simultaneously to more than one class up to a certain degree, which can not be effectively handled by PCA or LDA. Hence, adopting a feature projection method that maximizes the margins between the features belonging to different stages at each local area, while considering the fuzzy nature of drowsiness patterns, may lead to better separation of the different drowsiness levels. This motivates the proposed UFLPA. The proposed UFLPA also presents a set of mutually uncorrelated features, which is a desired property in any pattern recognition problem to minimize information redundancy [30].

\section{UNCORRELATED FUZZY LOCALITY PRESERVING ANALYSIS}

Given a universal set with elements $\mathbf{x}_{k}$ distributed in a pattern space as $\mathrm{X}=\left\{\mathbf{x}_{1}, \mathbf{x}_{2}, \ldots, \mathbf{x}_{l}\right\}$, where $k=1,2, \ldots, l$ with $l$ being the total number of patterns. For simplicity, it will be useful to describe the membership value that the $k$ th vector has in the $i$ th class with the following notation

$$
\mu_{i k}=\mu_{i}\left(\mathbf{x}_{k}\right) \in[0,1]
$$

Denote the mean of the data samples that belong to class $i$ as $\overline{\mathbf{x}_{i}}$ and the radius of the data as $r$

$$
r=\max \left\|\overline{\mathbf{x}}_{i}-\mathbf{x}_{k}\right\|_{\sigma}
$$

Then the fuzzy membership $\mu_{i k}$ can be calculated as follows

$$
\mu_{i k}=\left(\frac{\left\|\overline{\mathbf{x}}_{i}-\mathbf{x}_{k}\right\|}{r+\epsilon}\right)^{\frac{-2}{m-1}}
$$

where $m$ is the fuzzification parameter chosen as $m>1$, and $\epsilon>0$ is a small value to avoid singularity. Finally, the membership of each of the samples in all of the $c$-problem classes is normalized according to $\sum_{i=1}^{c} \mu_{i k}=1$.

The description of the proposed UFLPA proceeds with the fuzzy total scatter matrix given by Watada et al. [28] as:

$$
S_{T}=\sum_{i=1}^{c} \sum_{k=1}^{l_{i}} \mu_{i k}\left(\mathbf{x}_{k}-\overline{\mathbf{x}}\right)\left(\mathbf{x}_{k}-\overline{\mathbf{x}}\right)^{T}
$$

where $\mu_{i k}$ is the membership of pattern $k$ in class $i, \mathbf{x}_{k}$ is the $k^{\text {th }}$ sample, $l_{i}$ is the number of training samples for class $i, \overline{\mathbf{x}}$ is the mean of the training samples.

$$
\overline{\mathbf{x}}=\frac{\sum_{i=1}^{c} \sum_{k=1}^{l_{i}} \mu_{i k} \mathbf{x}_{k}}{\sum_{i=1}^{c} \sum_{k=1}^{l_{i}} \mu_{i k}}
$$

Unlike Watada et al. [28], which focused on the global structure of the data, we aim to modify Eq. 4 to focus on the local structure of the data. We proceed with the modification of the total scatter matrix starting from Eq.4 as follows 


$$
S_{T}=\sum_{i=1}^{c} \sum_{k=1}^{l_{i}} \mu_{i k}\left(\mathbf{x}_{k} \mathbf{x}_{k}^{T}-\overline{\mathbf{x}} \mathbf{x}_{k}^{T}-\mathbf{x}_{k} \overline{\mathbf{x}}^{T}+\overline{\mathbf{x x}}^{T}\right)
$$

Using Eq.5 we replace $\sum_{i=1}^{c} \sum_{k=1}^{l_{i}} \mu_{i k} \mathbf{x}_{k}^{T} \quad$ with $\overline{\mathbf{x}}^{T} \sum_{i=1}^{c} \sum_{k=1}^{l_{i}} \mu_{i k}$, and $\quad \sum_{i=1}^{c} \sum_{k=1}^{l_{i}} \mu_{i k} \mathbf{x}_{k} \quad$ with $\overline{\mathbf{x}} \sum_{i=1}^{c} \sum_{k=1}^{l_{i}} \mu_{i k}$. Thus Eq.6 can be re-written as

$$
\begin{aligned}
S_{T}= & \frac{1}{\sum_{i=1}^{c} \sum_{j=1}^{l_{i}} \mu_{i k}}\left[\sum_{i=1}^{c} \sum_{k=1}^{l_{i}} \sum_{p=1}^{c} \sum_{j=1}^{l_{p}} \mu_{i j} \mu_{p k} \mathbf{x}_{k} \mathbf{x}_{k}^{T}\right. \\
& \left.-\sum_{i=1}^{c} \sum_{k=1}^{l_{i}} \sum_{p=1}^{c} \sum_{j=1}^{l_{p}} \mu_{i j} \mu_{p k} \mathbf{x}_{k} \mathbf{x}_{j}^{T}\right]
\end{aligned}
$$

In order to simplify the above equation, we provide the following definition

Definition-1: $N$ is the fuzzy amount of elements in all fuzzy classes, and this is given by:

$$
N=\sum_{i=1}^{c} \sum_{k=1}^{l_{i}} \mu_{i k}
$$

Thus, by using the above definition in Eq.7 and with some linear algebra we end up with

$$
S_{T}=\frac{1}{2 N} \sum_{i=1}^{c} \sum_{k=1}^{l_{i}} \sum_{p=1}^{c} \sum_{j=1}^{l_{p}} \mu_{i j} \mu_{p k}\left(\mathbf{x}_{k}-\mathbf{x}_{j}\right)\left(\mathbf{x}_{k}-\mathbf{x}_{j}\right)^{T}
$$

The total scatter matrix is equal to the summation of the within-class scatter matrix and the between-class scatter matrix, that is

$$
S_{T}=S_{B}+S_{W}
$$

Thus, Eq. 9 can be decomposed into the following two equations

$$
\begin{aligned}
& S_{W}=\frac{1}{2 N} \sum_{i=p=1}^{c} \sum_{k=1}^{l_{i}} \sum_{j=1}^{l_{p}} \mu_{i k} \mu_{p j}\left(\mathbf{x}_{k}-\mathbf{x}_{j}\right)\left(\mathbf{x}_{k}-\mathbf{x}_{j}\right)^{T} \\
& S_{B}=\frac{1}{2 N} \sum_{\substack{i=1 \\
c}}^{c} \sum_{\substack{p=1 \\
p \neq i}}^{c} \sum_{k=1}^{l_{i}} \sum_{j=1}^{l_{p}} \mu_{i k} \mu_{p j}\left(\mathbf{x}_{k}-\mathbf{x}_{j}\right)\left(\mathbf{x}_{k}-\mathbf{x}_{j}\right)^{T}
\end{aligned}
$$

Thus, Eq. 11 indicates that the objective of the new $S_{W}$ is to minimize the distance between samples of the same class when projecting them, thus preserving the local neighborhood information. On the other hand, the proposed new $S_{B}$ maximizes the margin between data points from different classes at each local neighborhood. Additionally, both of $S_{W}$ and $S_{B}$ also incorporate the membership values to consider the samples contribution in the different class while preserving the local data structure. Thus, the above equations can be used to discover the discriminant structure in the data. However, in order to discover both geometrical and discriminant structures of the data manifold we adopt an approach from Cai et al. [25], in their Locality Sensitive Discriminant Analysis (LSDA) method, to construct two local neighborhood graphs to accurately characterize both of these structures. A linear transformation is then found to optimally preserve the local neighborhood information, as well as discriminant information using the notion of graph Laplacians.

Let $U_{w}$ and $U_{b}$ be the weight matrices for our proposed fuzzy within-class graph and the fuzzy between-class graph respectively, and let $N\left(\mathbf{x}_{k}\right)$ be the set of the nearest few neighbours to $\mathbf{x}_{k} . N\left(\mathbf{x}_{k}\right)$ can be split into $N_{w}\left(\mathbf{x}_{k}\right)$ (for neighbours sharing the same class label as $\left.\mathbf{x}_{k}\right)$ and $N_{b}\left(\mathbf{x}_{k}\right)$ (for neighbours sharing different class label to $\mathbf{x}_{k}$ ). We define $U_{w}$ and $U_{b}$ according to the following equations:

$$
\begin{aligned}
& U_{w, k j}=\left\{\begin{array}{ll}
\mu_{i k} \mu_{p j} & \text { if } \mathbf{x}_{j} \in N_{w}\left(\mathbf{x}_{k}\right) \\
0 & \text { if } \mathrm{p} \neq \mathrm{i}
\end{array}\right\} \\
& U_{b, k j}=\left\{\begin{array}{ll}
0 & \text { if } \mathrm{p}=\mathrm{i} \\
\mu_{i k} \mu_{p j} & \text { if } \mathbf{x}_{j} \in N_{b}\left(\mathbf{x}_{k}\right)
\end{array}\right\}
\end{aligned}
$$

The nature of the above matrices implies dropping the class label indices $i$ and $p$, as both matrices account for the total number of samples. This is done to facilitate using sparse implementations of these matrices to minimize the associated computational cost. However, before proceeding with the rest of the derivations, the differences between our approach and that of LSDA [25] should be clearly stated here.

- The first is that our approach utilizes fuzzy memberships when constructing the neighborhood graphs, while LSDA [25] puts an edge in the graph only if two samples from the same class are among the $k$ nearest neighbors of each other only.

- Additionally, we manipulate the terms in the final optimization problem to make the generated features statistically uncorrelated, which is not the case with LSDA.

Using Eq.13 and Eq.14, one can simplify Eq.11 into

$$
S_{W}=\frac{1}{2 N} \sum_{k, j}\left(\mathbf{x}_{k}-\mathbf{x}_{j}\right)\left(\mathbf{x}_{k}-\mathbf{x}_{j}\right)^{T} U_{w, k j}
$$

which simplifies to

$$
S_{W}=\frac{1}{N}\left(X D X^{T}-X U_{w} X^{T}\right)
$$

where $D$ is a diagonal matrix; its entries are column sums of $U_{w}$ (or row sums since $U_{w}$ is symmetric), $D_{k k}=$ $\sum_{j} U_{w, k j}$. In a similar manner to the above derivations, the between-class scatter matrix can also be reformulated as:

$$
S_{B}=\frac{1}{N}\left(X E X^{T}-X U_{b} X^{T}\right)
$$


where $E$ is a diagonal matrix; its entries are column (or row, since $U_{b}$ is symmetric) sums of $U_{b}, E_{k k}=\sum_{j} U_{b, k j}$.

The transformation matrix related to UFLPA can be acquired as the eigenvectors of the following equation assuming that the scatter matrices are not singular.

$$
G_{U F L P A}=\arg \max _{G} \operatorname{trace}\left(\frac{G^{T} S_{B} G}{G^{T} S_{W} G}\right),
$$

In our proposed UFLPA, the goal is to reduce the amount of information redundancy and produce a set of uncorrelated features while avoiding the singularity problem (if it exists). Thus, we propose to employ an approach based on simultaneous diagonalization of the matrices according to Ye et al. [31] to end up with a set of uncorrelated features. To proceed with this process, we start by modifying the above objective function.

A closer look at Eq.16 and Eq.17 reveals that Eq.18 can be maximized by minimizing $U_{b}$, i.e., minimize the memberships of the samples that belong to a certain class in all other classes. On the other hand, to minimize the denominator we need to maximize $U_{w}$, i.e., maximize the memberships of the samples that belong to a certain class in the same class. To proceed with further analysis, we employ a normalized Laplacian graph i.e., replace $L_{w}=D-U_{w}$ by $L_{w}=I-(\sqrt{D})^{-1} U_{w}(\sqrt{D})^{-1}$, and $L_{b}=E-U_{b}$ by $L_{b}=I-(\sqrt{E})^{-1} U_{b}(\sqrt{E})^{-1}$. Additionally, we modify the numerator to $X\left(\alpha L_{b}+(1-\alpha)(\sqrt{D})^{-1} U_{w}(\sqrt{D})^{-1}\right) X^{T}$ where $\alpha$ is a suitable constant $(0 \leq \alpha \leq 1)$. Thus, the optimization problem reduces to

$$
\arg \max _{G} \operatorname{trace}\left(\frac{G^{T} X A X^{T} G}{G^{T} X X^{T} G}\right)
$$

where $A=\left(\alpha L_{b}+(1-\alpha)(\sqrt{D})^{-1} U_{w}(\sqrt{D})^{-1}\right)$. In order to solve the singularity problem, the SVD decomposition is employed here. Supposing $\operatorname{rank}(X)=r$, the SVD decomposition of $X$ is then given as $X=U \Sigma V^{T}$, where $U \in \Re^{n \times r}, \Sigma=\operatorname{diag}\left(\sigma_{1}, \ldots ., \sigma_{r}\right) \in \Re^{r \times r}$ with $\sigma_{1} \geq \ldots \geq \sigma_{r}$, and $V=\left[\mathbf{v}_{1}, \ldots \mathbf{v}_{r}\right] \in \Re^{l \times r}$. Then

$$
\arg \max _{G} \operatorname{trace}\left(\frac{G^{T} U \Sigma V^{T} A V \Sigma U^{T} G}{G^{T} U \Sigma V^{T} V \Sigma U^{T} G}\right)
$$

To simplify the above optimization problem a simple variable modification scheme is then utilized by setting $B=$ $\Sigma U^{T} G$ to get

$$
\arg \max _{B} \operatorname{trace}\left(\frac{B^{T} V^{T} A V B}{B^{T} B}\right)
$$

Thus, the eigenvectors of the original problem can now be found by finding first the eigenvectors of Eq.21, represented by the columns of $B^{*}$. After getting $B^{*}$, the $G^{*}$ can be found by simply taking $G^{*}=U \Sigma^{-1} B^{*}$ and the extracted features in this case are uncorrelated (the reader may refer to [27], [31] for the proof).

\section{Data Collection Procedure}

Thirty-one subjects (volunteer drivers, all males) aged between 20-69 years were recruited to perform a driving simulation task. All participants gave written consent for the study, which was approved by the institutional ethical committee. To qualify for the study, a lifestyle appraisal questionnaire was used requiring participants to have no medical contraindications such as severe concomitant disease, alcoholism, drug abuse, and psychological or intellectual problems likely to limit compliance [32]. Most of the studies were conducted between 9:00AM to 1:00PM with the total study time involving 2-3 hours per subject. This included completing questionnaires, physiological sensor attachment and performing the driving simulator task. Caffeine, tea or food as well as smoking were restricted approximately $4 \mathrm{hr}$ and alcohol $24 \mathrm{hr}$ before the study.

The data collection process included two simultaneous collection procedures. The first was to collect the physiological changes associated with the drowsiness when performing a simulation task, while the second involved monitoring the driver facial expressions through a vision system. The driving simulator equipment consisted of a large display unit with in-built steering wheel, brakes, and accelerator. The video display showed the current speed, the driving environment, other cars, pedestrians, and other road stimuli. The participants were asked to breathe normally and restrict all unnecessary movements as much as possible during driving. The driving simulator software was acquired from Systems Technology, Inc. (STI), USA, for which the STISIM driver was utilized in this task. All subjects were given instructions on the operation of the simulator prior to the study. Two driving sessions were completed by each of the drivers. The initial driving session was approximately 25 min of alert driving, with a track involving many cars and stimuli on the road to serve as the baseline measure. The alert driving session was followed by monotonous driving session, in which participants were required to drive continuously for approximately $1 \mathrm{~h}$. This session involved the participants driving with very few road stimuli in a track resembling country-side driving.

Simultaneous physiological measurements were recorded during the driving sessions. A FlexComp Infiniti encoder, from Thought Technology Inc., was utilized as the physiological data acquisition device (data was sampled at 2048 $\mathrm{Hz}$ ). It consisted of three Electroencephalogram (EEG) channels, one Electrooculogram (EOG) channel, and one Electrocardiogram (ECG channel. The three EEG channels were recorded according to the 10-20 international standard of electrode placement [33], with EEG electrodes placed at $\mathrm{Fz}$ (frontal), T8 (temporal), and $\mathrm{Oz}$ (occipital) sites. A monopolar montage was used, that is, EEG activity was recorded in relation to a linked-ear reference. Vertical EOG was recorded from the left eye, and later used to identify drowsiness by observing the blink rate of each subject. A surface electrode measuring the ECG signal was utilized with the reference electrode placed on the shoulder and the 
active electrodes placed on the right and the left chest. Blood pressure and heart rate were collected before and after the driving task.

Physical signs of drowsiness were identified using video images of the driver's face, linked in real time with the physiological measurements. The level of drowsiness was estimated based on the Wierewille and Ellsworth criteria [34]. Specific facial features characterized the drowsiness level observed during the driving task, including the facial tone, slow eyelid closure, and mannerisms (rubbing, yawning, nodding, etc.). A continuous drowsiness scale containing five descriptors was utilized. These descriptors are given as: Alert-or Not Drowsy (class-1), Slightly Drowsy (class-2), Moderately Drowsy (class-3), Significantly Drowsy (class4), and Extremely Drowsy (class-5) [34]. Three observers, trained by a neuroscientist, visually rated the total driving time in video segments of one minute duration and assigned a corresponding drowsiness descriptor according to the aforementioned scale. In order to form the final class label for each subject's data segments, a majority voting process was utilized. In such a process, each segment is assigned the label that most of the observers agreed on. The final label of the scored segments was considered to be the class label for the stages of drowsiness required to train any classification system.

\section{EXPERIMENTS AND RESULTS}

The first step in the conducted experiments was to extract a set of features from the available 5 channels (3 EEG plus 1 ECG plus 1 EOG) utilized in this research. A windowing approach was utilized in which a sliding window of $10 \mathrm{sec}$ length was incremented by $2 \mathrm{sec}$ each time on each of the channels. Given that there is no one agreed-upon feature extraction method in the literature, a variety of features were extracted from each of the windows. Specifically, the extracted feature set included: number of zero crossings (1 feature), Hjorth parameters (3 features), root mean square (1 feature), autoregressive model coefficients (10 features), the spectral moments (4 features), waveform length ( 1 feature), and Barlow parameters (3 features) (most available from the BioSig toolbox at http://biosig. sourceforge. net/). Thus, the extracted feature set is made of a total of 115 features (23 feature/channel $\times 5$ channels $=115$ features). For simplicity we denote this feature set as the TDAR, i.e., a combination of Time Domain AutoRegressive features.

In order to test the performance of the proposed UFLPA against the available methods from the literature, all of the following methods were implemented: LPP [26], LSDA [25] (all implementations with regularization are available from http://www.cs.uiuc. edu/homes/dengcai2/Data/data.html), the Uncorrelated Linear Discriminant Analysis (ULDA) [31] (available from http://www.public.asu.edu/ jye02/ Software/index.html), the Fuzzy Linear Discriminant Analysis (FLDA) [28], [29], in addition to the classification accuracy of the Baseline, i.e., using the total number of features without reduction. Two classifiers were utilized in the experiments: the first is the well-known $k$-Nearest Neighbor ( $k \mathrm{NN}$, with $k=1$ providing powerful enough results), and the support vector machine classifier (the LIBSVM implementation available at http://www.csie.ntu.edu.tw/ c cjlin/libsvm/).

The average classification error results across all of the 31 subjects using the $k \mathrm{NN}$ and LIBSVM classifiers are shown in Fig.1. It clearly shows the superiority of the proposed UFLPA when comparing with all other feature projection methods. Additionally, it can be seen that more features provided better classification results in UFLPA than that of KNNFLDA or ULDA. Maximum of 30 features were considered in this experiment for which the UFLPA managed to achieve an average of $\approx 94 \%$ with the LIBSVM classifier and $\approx 95 \%$ with the $k \mathrm{NN}$ classifier across all subjects.

It was noted that the drivers exhibited different levels of drowsiness during the simulation test. Therefore we have analyzed the average classification errors for different groups having drowsiness levels of 2, 3, 4 and 5 (Fig.2). The error bars represent the standard deviation.

All of these results indicate that the proposed UFLPA managed to achieve higher classification accuracy rates with a fewer number of features than all other methods. It could also be noted that the accuracies of UFLPA, LSDA, and LPP were higher when using the $k \mathrm{NN}$ classifier than that of the LIBSVM classifier. It can be justified by the fact that the objective function of these methods in the minimization work well with the $k \mathrm{NN}$ as $k \mathrm{NN}$ also focuses on distances between each sample and its neighbors. On the other hand, the performances of ULDA and KNNFLDA were more powerful with the LIBSVM classifier than with the $k \mathrm{NN}$ classifier, as such methods focus on preserving the global Euclidean structure rather than the local manifold structure. However, for the specific application of drowsiness levels classification the local manifold structure is more important than the global structure, as proved by the classification results. Additionally, one can clearly notice that the use of discriminant analysis methods that generate $c-1$ features generally have poor performances in this application.

In order to prove the statistical significance of the achieved classification results by UFLPA across all 31 subjects, a twoway analysis of variance (ANOVA) test with significance level set to $\rho=0.05$ was utilized. The ANOVA test results proved the statistical significance of the results achieved by UFLPA in comparison to all other methods, as the achieved $\rho$ was always smaller than 0.001 .

The second adopted significance measure was to look at the confusion matrices (class-wise classification accuracies). These were computed as an average for all of the 31 subjects across 30 runs. The mean of the resultant average confusion matrix with each feature projection method and classifier was then plotted as shown in Fig.3 with error bars representing standard deviations. These results indicate again the superiority of the results achieved by the proposed UFLPA in comparison to the other methods. 


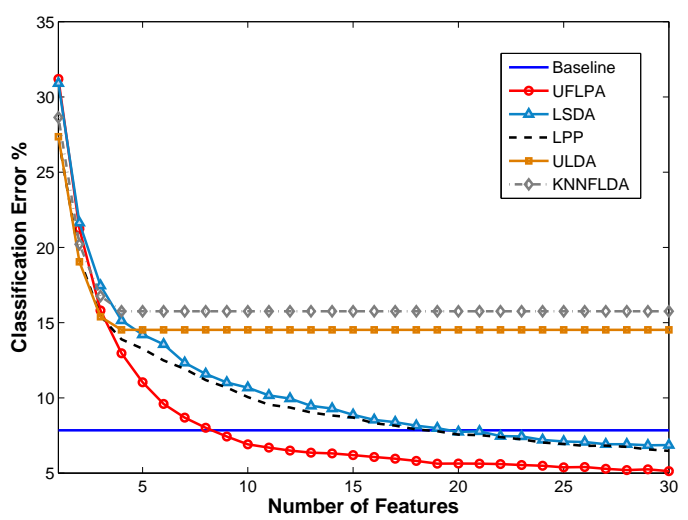

(a) Using $k \mathrm{NN}$ classifier

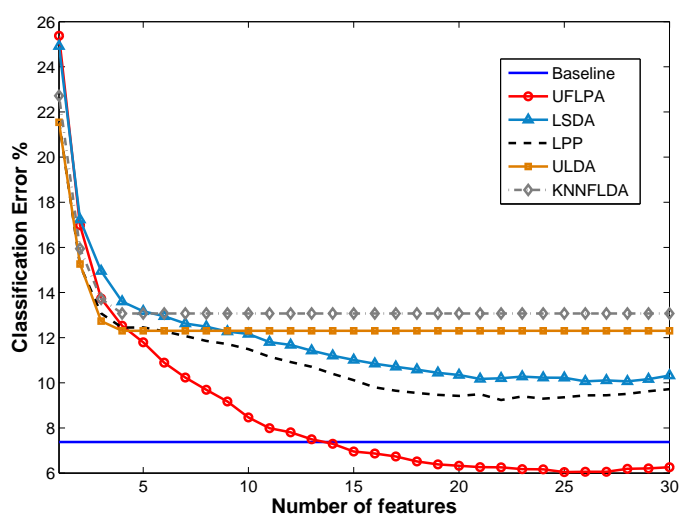

(b) Using LIBSVM classifier

Fig. 1. Classification results using different feature projection methods with two different classifiers averaged across 31 subjects

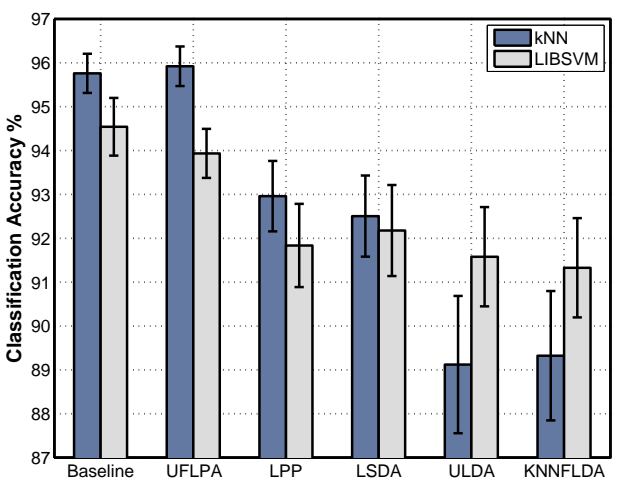

(a) Average across 3 subjects exhibiting 2 drowsiness levels

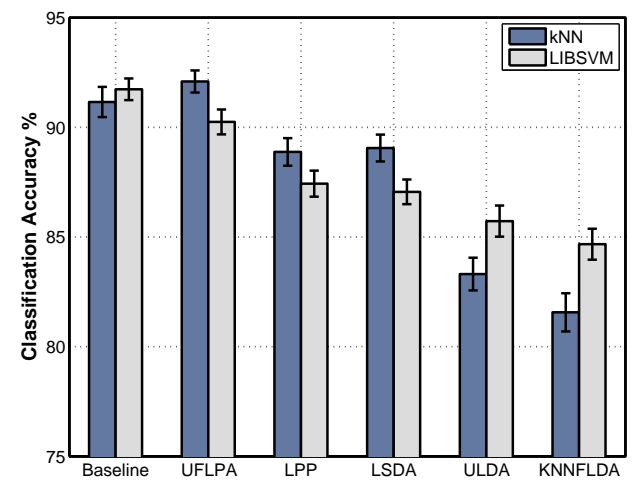

(c) Average across 10 subjects exhibiting 4 drowsiness levels

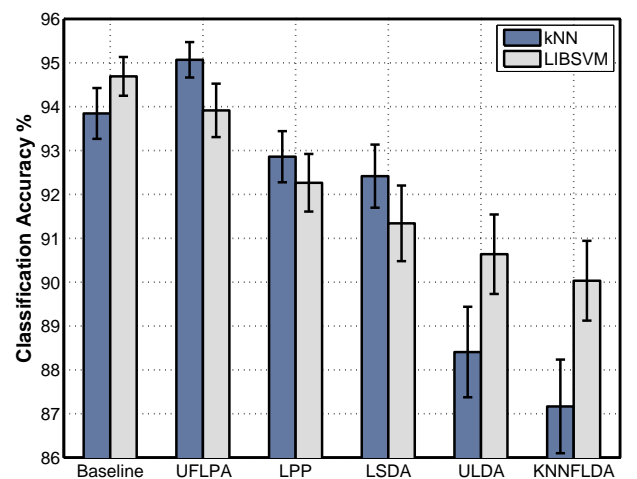

(b) Average across 12 subjects exhibiting 3 drowsiness levels

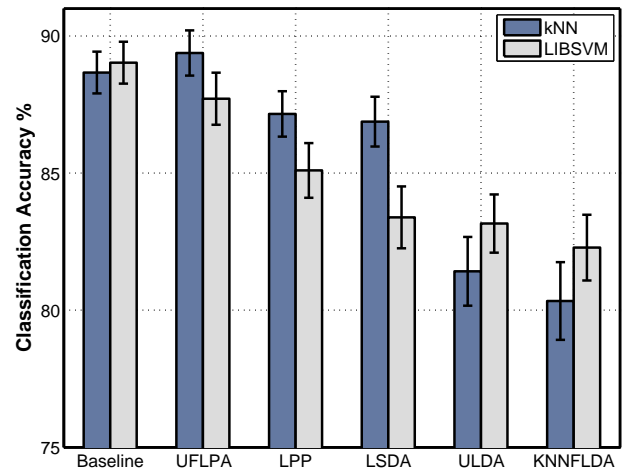

(d) Average across 6 subjects exhibiting 5 drowsiness levels

Fig. 2. Classification results using different feature projection methods with two different classifiers averaged across 31 subjects

\section{CONCLUSION}

A new feature projection and dimensionality reduction method was proposed in this paper. The UFLPA method aims at preserving the local structure of the data samples while considering the contribution of each sample in the different drowsiness levels. Additionally, the extracted features by
UFLPA were made to be statistically uncorrelated between each other, thus minimizing the amount of information redundancy. The performance of the proposed UFLPA was tested on datasets collected from 31 drivers while performing a simulation test. Different drowsiness levels were exhibited by each of the drivers and the proposed UFLPA was able to show better performance than other methods from the 


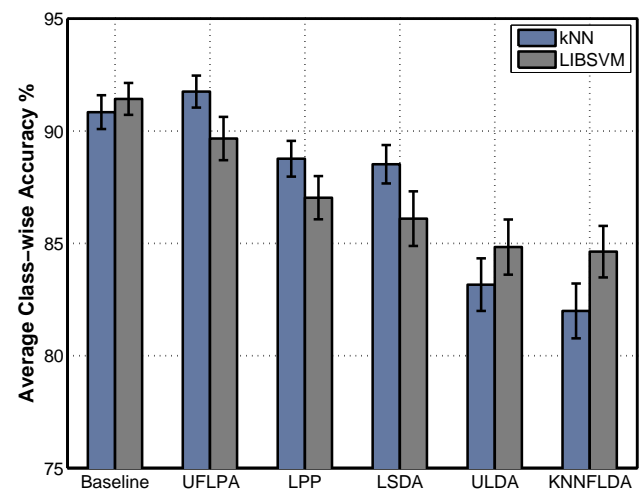

Fig. 3. Average of class-wise accuracy across thirty-one subjects using different classifiers with error bars indicating standard deviations.

literature in classifying these drowsiness levels, achieving an average of $\approx 95 \%$ using the $k \mathrm{NN}$ classifier, and an average of $\approx 94 \%$ with the LIBSVM classifier, across all of the 31 drivers while using only 30 features.

\section{REFERENCES}

[1] C. T. Lin, L. W. Ko, and T. K. Shen, "Computational intelligent brain computer interface and its applications on driving cognition", IEEE Computational Intelligence Magazine, pp. 32-46, 2009.

[2] H. Ueno, M. Kaneda, and M. Tsukino, "Development of drowsiness detection system", in Proceedings of Vehicle Navigation and Information Systems Conference, vol. 31, pp. 15-20, 1994.

[3] M. Eriksson and N. P. Papanikotopoulos, "Eye-tracking for detection of driver fatigue", in Proceedings of the IEEE Conference on Intelligent Transportation Systems, pp. 314-319, 1997.

[4] T. A. Dingus, H. L. Hardee and W. W. Wierwille, "Developement of models for on-board detection of driver impairment", Accident Analysis and Prevention, vol. 19, no. 4, pp. 271-283, 1987.

[5] R. W. Allen, Z. Parseghian, S. Kelly, and T. J. Rosenthal, "An experimental study of driver alertness monitoring", National Highway Traffic Safety Administration, Office of Crash Avoidance Research, paper no. 508, 1994.

[6] Q. Ji, Z. Zhu, and P. Lan, "Real-time nonintrusive monitoring and prediction of driver fatigue", IEEE Transactions on Vechicular Technology, vol. 53, no. 4, pp. 1052-1068, 2004.

[7] C. T. Lin, R. C Wu, S. F. Liang, W. H. Chao, Y. J. Chen, and T. P. Jung, "EEG-Based drowsiness estimation for safety driving using independent component analysis", IEEE Transactions on Circuits and Systems-I, vol. 25, no. 12, pp. 2726-2738, 2005.

[8] I. D. Brown, "Car driving and fatigue", Triangle Sandoz Journal of Medical Services, vol.8, pp 131-137, 1967.

[9] S. k. 1. Lal and A. Craig, " critical review of the psychophysiology of driver fatigue", Biological Psychology, vol. 55, pp. 173-194, 2001.

[10] E, Zilberg, Z. M. Xu, D. Burton, M. Karrar, and S. Lal, ”Statistical validation of physiological indicators for noninvasive and hybrid drowsiness detection system", African journal of information and Communication Technology, vol. 5, no. 2, pp. 75-83, 2009.

[11] S. K. L. Lal and A. Craig, "Psychological effects associated with drowsiness: Driver fatigue and Electroencephalography", International Journal of Psychophysiology, vol. 55, pp. 183-189, 2001.

[12] T. Akerstedt, G. Kecklund, and A. Knutsson, "Manifest sleepiness and the spectral content of the EEG during shift work", Sleep, vol. 14. no. 3, pp. 221-225, 1991.

[13] B. T. Jap, S. Lal, P. Fischer, and E. Bekiaris, "Using EEG spectral components to assess algorithms for detecting fatigue", Expert Systems with Applications, vol. 36, pp.2352-2359, 2009.

[14] H. Shuyan and Z. Gangtie, "Driver drowsiness detection with eyelid related parameters by Support Vector Machine", Expert Systems with Applications, vol. 36, pp. 7651-7658, 2009.
[15] A. Tsuchida, M. S. Bhuiyan, and K. Oguri, "Estimation of drowsiness level based on Eyelid closure and heart rate variability", In Proceedings of the 31st Annual International Conference of the IEEE EMBS, pp. 2543-2546, 2009

[16] J. W. Fu, M. Li, and B. L. Lu, ”Detecting drowsiness in driving simulation based on EEG", In Proceedings of the 8th International Workshop of Autonomous Systems-Self-Organization, Management, and Control, October 67, 2008.

[17] C. T. Lin, L. W. Ko, I. F. Chung, T. Y. Huang, Y. C. Chen, T. P. Jung, and S. F. Liang, "Adaptive EEG-based Alertness estimation system by using ICA-Based Fuzzy Neural Networks", IEEE Transactions on Circuits and Systems-I, vol. 53, no. 11, pp. 2469-2476, 2006.

[18] J. Wallerius, L. J. Trejo, R. Matthews, R. Rosipal, and J. A. Caldwell, "Robust feature extraction and classification of EEG spectra for real-time classification of cognitive state", In Proceedings of 11th International Conference on Human Computer Interaction, Las Vegas, NV, pp. 1-10, 2005.

[19] M. V. M. Yeo, X. Li, K. Shen, E. P.V Wilder-Smith, "Can SVM be used for automatic EEG detection of drowsiness during car driving ?", Safety Science, vol. 47, pp. 115-124, 2009.

[20] C. Zhang, C. X. Zheng, and X. L. Yu, "Automatic recognition of cognitive and fatigue from physiological indices by using wavelet packet transform and kernel learning methods", Expert Systems with Applications, vol. 26, pp. 4664-4671, 2009.

[21] D. L. Swets and J. Weng, "Using discriminant eigenfeatures for image retrieval", IEEE Transactions on Pattern Analysis and Machine Intelligence, vol. 18, no. 8, pp. 831-836, 1996.

[22] P. N. Belhumeur, J. P. Hepanha, and D. J. Kriegman, "Eigenfaces vs Fisherfaces: recognition using class specific linear projection", IEEE Transactions on Pattern Analysis and Machine Intelligence, vol. 19, no. 7, pp. 711-720, 1997.

[23] K. Torkkola, "Linear discriminant analysis in document classification", In Proceedings of the IEEE International Conference on Data Mining, Workshop on Text Mining, 2001.

[24] J. H. Friedman, "Regularized Discriminant Analysis", Journal of the American Statistical Association, vol. 84, no. 405, pp. 165-175, 1989.

[25] D. Cai and X. He and K. Zhou and J. Han and H. Bao, "Locality Sensitive Discriminant Analysis", Proceeding of the International Joint Conference on Artificial Intelligence (IJCAI'07), Hyderabad, India, 2007.

[26] X. He and P. Niyogi, "Locality Preserving Projections", Advances in Neural Information Processing Systems 16, Vancouver, Canada, 2003.

[27] D. Cai, X. He, and J. Han, "SRDA: An Efficient Algorithm for Large Scale Discriminant Analysis", IEEE Transactions on Knowledge and Data Engineering, vol. 20, no. 1, pp. 1-12, 2008.

[28] J. Watada, H. Tanaka, and K. Asai, "Fuzzy discriminant analysis in fuzzy groups", Fuzzy Sets and Systems, vol. 19, no. 3, pp. 261-271, 1986.

[29] Z. P. Chen, J. H. Jiang, Y. Li, Y. Z. Liang, and R. Q. Yu, ”Fuzzy linear discriminant analysis for chemical data sets", Chemometrics and Intelligent Laboratory Systems, vol. 45, no. 1-2, pp. 295-302, 1999.

[30] J. Ye, "Characterization of a Family of Algorithms for Generalized Discriminant Analysis on Undersampled Problems", Journal of Machine Learning Research, vol. 6, no. , pp. 483-502, 2005.

[31] J. Ye, R. Janardan, Q. Li, and H. Park, "Feature Reduction via Generalized Uncorrelated Linear Discriminant Analysis", IEEE Transactions on Knowledge and Data Engineering, vol. 18, no. 10, pp. 1312-1322, 2006.

[32] A. Craig, K. Hancock, and M. Craig, "The Lifestyle Appraisal Questionnaire: A comprehensive assessment of health and stress", Psychology and Health, vol. 11, pp. 331-343, 1996.

[33] H. H. Jasper, "The ten-twenty electrode system of the International Federation", Electroencephalography and Clinical Neurophysiology, vol. 10 , pp. 371-375, 1958.

[34] W. W. Wierwille and L. A. Ellsworth, "Evaluation of driver drowsiness by trained raters", Accident Analysis and Prevention, vol. 26, no. 5, pp. 571-581, 1994. 